\title{
Investigation of the Inlet and Outlet Location on the Soundproofing Ventilation Unit
}

\author{
Nasu Tsunetoshi ${ }^{1}$ and Nishimura Tsuyoshi ${ }^{*}, 2$ \\ ${ }^{1}$ Faculty of Engineering, Kumamoto University, 2-39-1 Kurokami Kumamoto, Japan \\ ${ }^{2}$ Faculty of Computer Science, Sojo University, 4-22-1 Ikeda Kumamoto, Japan
}

\begin{abstract}
This paper deals with A-type and B-type rectangular cuboid SVU (soundproofing ventilation unit) contain an inlet and outlet in opposite surfaces. The difference between them is whether they are located in small or big cross section area. Following the theoretical calculation, the shape and location of the inlet and the outlet are determined by an investigation of the distribution of higher order mode waves formed inside the SVU. Finally, experimental results of Atype and B-type based on the reverberation chamber method are shown to be in reasonable agreement with our theoretical predictions. The results of theoretical calculation and experiments conducted lead to the conclusion that A-type is suitable for soundproof ventilation unit.
\end{abstract}

Keywords: Higher order mode, resonance frequency, soundproofing, wave equation.

\section{INTRODUCTION}

The authors are presenting a concept of manufacturing windows called SPCW (Sound Proofing Casement Windows) which are capable of ventilating, regulating sunlight and reducing traffic noise inside the house of developing countries in tropical climate zones [1]. The size and the structure of SPCW are designed to be identical to the current casement windows in order to avoid any costs incurred when replacing those windows. The proposed SPCW combines two basic components namely the ventilation soundproofing unit and the lighting unit as shown in Fig. (1). The lighting unit can be constructed using one glass layer mounted between two soundproofing ventilation units (SVU). Due to the fact that SVU must have a large volume to attenuate the low frequency range of traffic noise, sound propagating through SVU is a combination of a plane wave and the higher order mode wave. The higher order mode wave has been reviewed in numerous experimental and analytical studies [3-10], particularly in the analysis and design of silencing systems. It generates very high levels of sound pressure, especially those at resonance frequencies. Therefore, in order to maximize the soundproof capability it is necessary to reduce the sound pressure levels or prevent the generation of higher order mode waves in any technique.

The SVU can consist of a square, rectangular cuboid or more complicated shapes depending on decorative considerations. Needless to say, the unit requires a simple internal structure and large input and output to maximize ventilation as well as preventing outside noise from entering the house. Actually, an input and output can be located at various positions on the SVU according to the design of window.

*Address correspondence to this author at the 4-22-1 Ikeda, Nishi-ku, Kumamoto 860-082, Sojo University, Japan; Tel: +81-96-326-3605; Fax: +81-96-326-3605; E-mails: nisimura@cis.sojo-u.ac.jp,nis0626@gmail.com

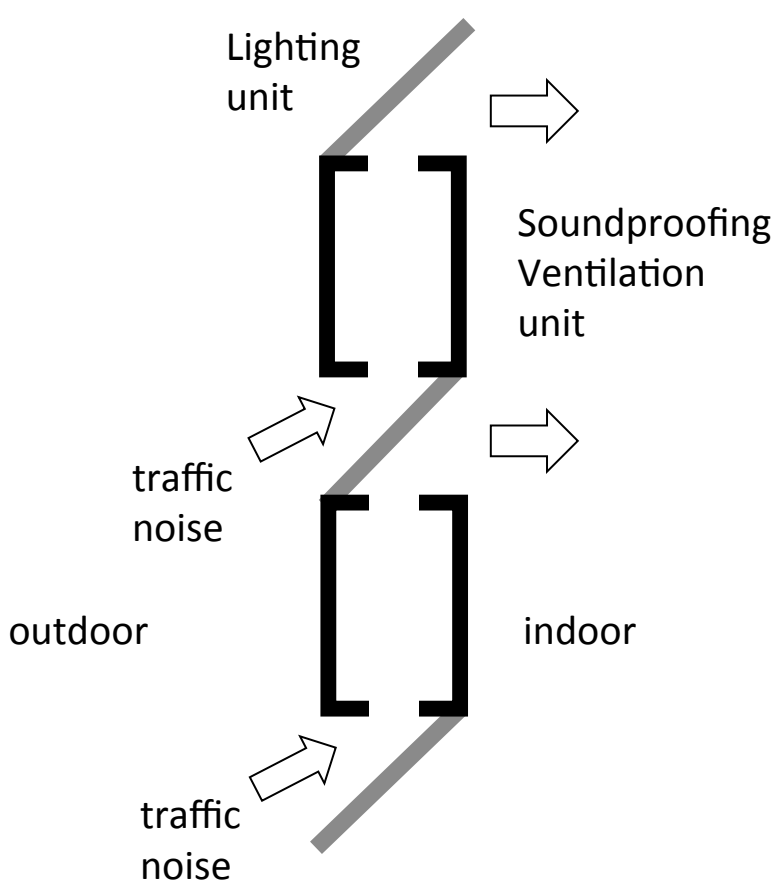

Fig. (1). A side view of proposed casement windows.

This paper deals with the rectangular cuboid SVU which contains an inlet and outlet in opposite surfaces as shown in Figs. $(2, \mathbf{3})$. The difference between them is whether they are located in small or big cross section area. Hereafter, we shall refer to them as A-type and B-type, respectively. Following the development of the theoretical calculation in section 2 , the shape and location of the inlet and the outlet are determined by an investigation of the distribution of higher order mode waves formed inside the SVU. Finally, experimental results of A-type and B-type based on the reverberation chamber method are shown to be in reasonable agreement with our theoretical predictions. 


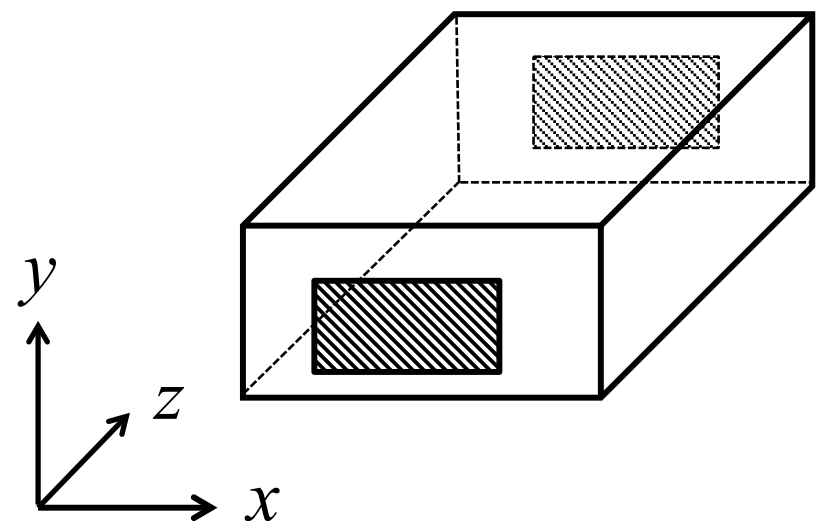

Fig. (2). A-type SVU.

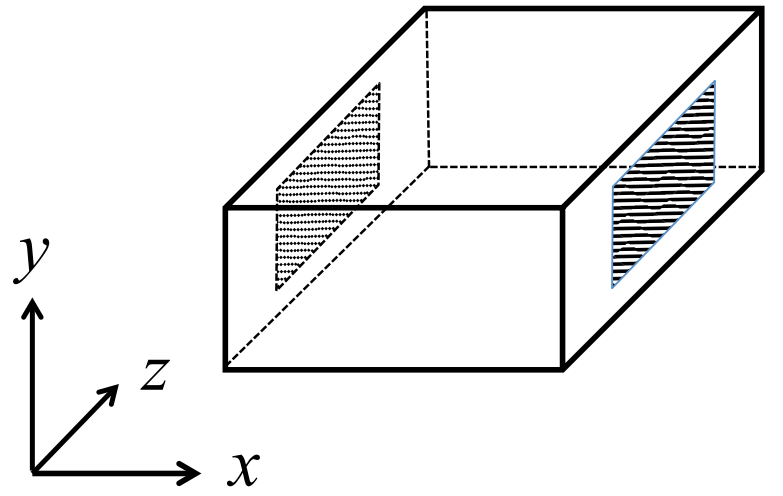

Fig. (3). B-type SVU.

\section{THEORETICAL CALCULATIONS}

Theoretical calculation of the sound pressure inside the rectangular cuboid including the effects of higher-order mode waves [2] is summarized as follows:

Consider the case of rectangular cuboid having a crosssectional area $S_{w}=a \times b$ and depth $L$ which has the inlet and outlet located on the opposite faces. The cross-sectional area of an inlet and outlet is $S_{0}=a_{0} \times b_{0}$ and $S_{L}=a_{L} \times b_{L}$, respectively.

The general solution of wave equation in terms of velocity potential can be given as:

$$
\begin{aligned}
& \phi=\left(A e^{\mu z}+B e^{-\mu z}\right)(C \sin \alpha x+D \cos \alpha x) \\
& \left(E \sin \sqrt{s^{2}-\alpha^{2}} y+F \cos \sqrt{s^{2}-\alpha^{2}} y\right)
\end{aligned}
$$

where $A, B, C, D, E$ and $\mathrm{F}$ are arbitrary constants determinable from the boundary conditions, other symbols are constants.

$V_{x}=-\partial \phi / \partial x, V_{y}=-\partial \phi / \partial y$ and $V_{z}=-\partial \phi / \partial z$ are the velocity components in the $\mathrm{x}, \mathrm{y}$ and $\mathrm{z}$ directions, respectively. Assuming the walls of the cavity to be perfectly rigid and the loss at the wall can be neglected, then boundary conditions may be expressed as:

(1) at $x=0, V_{x}=0$
(2) at $x=a, V_{x}=0$

(3) at $y=0, V_{y}=0$

(4) at $y=b, V_{y}=0$

(5) at $z=0, V_{z}=V_{0} F_{0}(x, y)$

(6) at $z=L, V_{z}=V_{L} F_{L}(x, y)$

where $V_{0}$ and $V_{L}$ are the driving velocity at the inlet and outlet, $F_{0}(x, y)=1$ at the inlet and $F_{0}(x, y)=0$ elsewhere, $F_{L}(x, y)=1$ at the outlet and $F_{L}(x, y)=0$ elsewhere.

According to the above boundary conditions, the velocity potential $\phi(x, y, z)$ can be determined as:

$$
\left.\begin{array}{l}
\phi(x, y, z)=\sum_{m=0}^{\infty} \sum_{n=0}^{\infty}\left\{\begin{array}{l}
\frac{\cosh \left(\mu_{m, n}(z-L)\right)}{\mu_{m, n} \sinh \left(\mu_{m, n} L\right)} U_{0} D_{m, n}^{a}+ \\
\frac{\cosh \left(\mu_{m, n} z\right)}{\mu_{m, n} \sinh \left(\mu_{m, n} L\right)} U_{L} D_{m, n}^{b}
\end{array}\right\} \\
\cos \left(\frac{m \pi x}{a}\right) \cos \left(\frac{n \pi y}{b}\right)
\end{array}\right\}
$$

Then the average sound pressure acting on the outlet can be obtained as:

$$
\begin{aligned}
& \bar{P}_{L}=\frac{1}{S_{L}} \int_{a_{L 0}}^{a_{L 1}} \int_{b_{L 0}}^{b_{L 1}} j k \rho c \phi(x, y, L) d x d y \\
& =j k Z_{w} \sum_{m=0}^{\infty} \sum_{n=0}^{\infty}\left\{\frac{\bar{Q}_{m, n}^{a} U_{0}}{\mu_{m, n} \sinh \left(\mu_{m, n} L\right)}+\frac{\cosh \left(\mu_{m, n} L\right) \bar{Q}_{m, n}^{b} U_{L}}{\mu_{m, n} \sinh \left(\mu_{m, n} L\right)}\right\}
\end{aligned}
$$

Moreover, expanding the above equation corresponding to variation of $m$ and $n$, we have:

$$
\begin{aligned}
& \bar{P}_{L}=j Z_{w}\left\{\begin{array}{c}
-\frac{U_{0}-U_{L} \cos (k L)}{\sin (k L)} \\
+\sum_{m=1}^{\infty}\left[\frac{\bar{Q}_{m, 0}^{a} U_{0}}{\mu_{m, 0} \sinh \left(\mu_{m, 0} L\right)}+\frac{\cosh \left(\mu_{m, 0} L\right) \bar{Q}_{m, 0}^{b} U_{L}}{\mu_{m, 0} \sinh \left(\mu_{m, 0} L\right)}\right] \\
+\sum_{n=1}^{\infty}\left[\frac{\bar{Q}_{0, n}^{a} U_{0}}{\mu_{0, n} \sinh \left(\mu_{0, n} L\right)}+\frac{\cosh \left(\mu_{0, n} L\right) \bar{Q}_{0, n}^{b} U_{L}}{\mu_{0, n} \sinh \left(\mu_{0, n} L\right)}\right] \\
+\sum_{\bullet}^{\dot{S}}\left[\frac{\bar{Q}_{m, n}^{a} U_{0}}{\mu_{m, n} \sinh \left(\mu_{m, n} L\right)}+\frac{\cosh \left(\mu_{m, n} L\right) \bar{Q}_{m, n}^{b} U_{L}}{\mu_{0, n} \sinh \left(\mu_{m, n} L\right)}\right]
\end{array}\right\}
\end{aligned}
$$

where

$$
\mu_{m, n}=\sqrt{\left(\frac{m \pi}{a}\right)^{2}+\left(\frac{n \pi}{b}\right)^{2}-k^{2}}
$$




$$
\begin{aligned}
& \bar{Q}_{0, n}^{a}=\frac{4}{(n \pi)^{2}}\left(\frac{b}{b_{01}-b_{00}}\right)\left(\frac{b}{b_{L 1}-b_{L 0}}\right)\left[\sin \left(\frac{n \pi b_{01}}{b}\right)-\sin \left(\frac{n \pi b_{00}}{b}\right)\right] \\
& {\left[\sin \left(\frac{n \pi b_{L 1}}{b}\right)-\sin \left(\frac{n \pi b_{L 0}}{b}\right)\right]} \\
& \bar{Q}_{m, 0}^{a}=\frac{4}{(m \pi)^{2}}\left(\frac{a}{a_{01}-a_{00}}\right)\left(\frac{a}{a_{L 1}-a_{L 0}}\right) \\
& {\left[\sin \left(\frac{m \pi a_{01}}{a}\right)-\sin \left(\frac{m \pi a_{00}}{a}\right)\right]\left[\sin \left(\frac{m \pi a_{L 1}}{a}\right)-\sin \left(\frac{m \pi a_{L 0}}{a}\right)\right]} \\
& \bar{Q}_{m, n}^{a}=\left(\frac{2 S_{w}}{m n \pi^{2}}\right)^{2} \frac{1}{S_{0} S_{L}}\left[\sin \left(\frac{m \pi a_{01}}{a}\right)-\sin \left(\frac{m \pi a_{00}}{a}\right)\right] \\
& {\left[\sin \left(\frac{n \pi b_{01}}{b}\right)-\sin \left(\frac{n \pi b_{00}}{b}\right)\right]} \\
& {\left[\sin \left(\frac{m \pi a_{L 1}}{a}\right)-\sin \left(\frac{m \pi a_{L 0}}{a}\right)\right]\left[\sin \left(\frac{n \pi b_{L 1}}{b}\right)-\sin \left(\frac{n \pi b_{L 0}}{b}\right)\right]} \\
& \bar{Q}_{0, n}^{b}=\frac{4}{(n \pi)^{2}}\left(\frac{b}{b_{L 1}-b_{L 0}}\right)^{2}\left[\sin \left(\frac{n \pi b_{L 1}}{b}\right)-\sin \left(\frac{n \pi b_{L 0}}{b}\right)\right]^{2} \\
& \bar{Q}_{m, 0}^{b}=\frac{4}{(m \pi)^{2}}\left(\frac{a}{a_{L 1}-a_{L 0}}\right)^{2}\left[\sin \left(\frac{m \pi a_{L 1}}{a}\right)-\sin \left(\frac{m \pi a_{L 0}}{a}\right)\right]^{2} \\
& \bar{Q}_{m, n}^{b}=\left(\frac{2 S_{w}}{m n \pi^{2}}\right)^{2} \frac{1}{S_{L}^{2}}\left[\sin \left(\frac{m \pi a_{L 1}}{a}\right)-\sin \left(\frac{m \pi a_{L 0}}{a}\right)\right]^{2} \\
& {\left[\sin \left(\frac{n \pi b_{L 1}}{b}\right)-\sin \left(\frac{n \pi b_{L 0}}{b}\right)\right]^{2}}
\end{aligned}
$$

$k$ is the wave number, $U_{0}$ and $U_{L}$ are the volume velocity at the inlet and outlet, the symbol $\dot{\sum}$ means $\sum_{m=0}^{\infty} \sum_{n=0}^{\infty}$ without the cases of $m$ and $n$ in 1st - 3rd terms of Eq. (10).

The first term in the bracket of Eq. (10) represents the sound pressure of the plane wave and the second one represents the sound pressure components of the higherorder mode waves, respectively. The output pressure $\bar{P}_{L}$ increases when its denominator $\sin (k \mathrm{~L})$ and $\mu_{m, n} \sinh \left(\mu_{m, n} L\right)$ are zero. Namely, at the following resonance frequencies of

$$
\begin{aligned}
& \sin (k L)=0 \quad \therefore \quad f=\eta \frac{c}{2 L} \quad(\eta=1,2,3 \ldots) \\
& \mu_{m, n} \sinh \left(\mu_{m, n} L\right)=0 \quad \therefore \\
& f_{m, n}=\frac{c}{2 \pi} \sqrt{\left(\frac{m \pi}{a}\right)^{2}+\left(\frac{n \pi}{b}\right)^{2}-\left(\frac{\eta \pi}{L}\right)^{2}} \quad(\eta=0,1,2,3 \ldots)
\end{aligned}
$$

\section{IMPROVEMENT FOR THE SOUNDPROOF EFFECT}

Generation mechanism of the frequencies of plane wave and the higher order mode wave can be understood according to the calculation example shown in Fig. (4). The curve of Eq. (18) and the sound pressure level of those wave components in $\mathrm{dB}$ are shown in Fig. (4a). Similarly, the curve of Eq. (19) and the sound pressure levels of $(2,0)$, (4, 0 ) higher order modes wave components in $\mathrm{dB}$ are shown in Fig. (4b). The plane wave and the higher order mode waves also have many resonance frequencies which occurred corresponding to the increasing value of in Eqs.(18) and (19). As evident from the results, the level of the latter is significantly greater than those of the former. Therefore, it is clear that when we are able to reduce an arbitrary higherorder wave mode, we will not only avoid resonance generated by this mode but also obtain a low level of the entire outlet sound pressure.

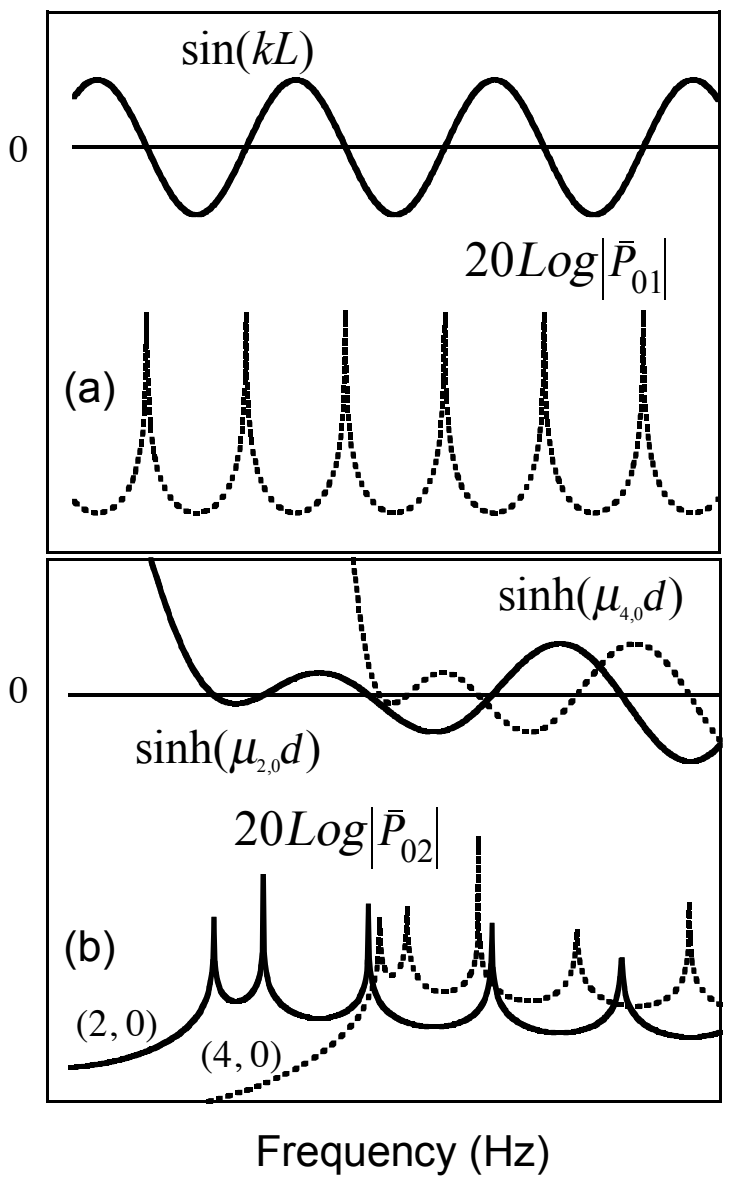

Fig. (4). The generation mechanism of sound pressure in B-type SVU having a dimension of $\mathrm{a}=0.48 \mathrm{~m}, \mathrm{~b}=0.075 \mathrm{~m}$ and $\mathrm{L}=0.29 \mathrm{~m}$. (a) Plane wave component defined by Eq. (10) (b) Higher order modes component defined by Eq. (10).

Fig. (5) shows the first resonance frequency of $(m, n)$ higher-order modes occur in A-type SVU. Fig. (6) shows those of B-type SVU and the comparison between two types of SVU. Note that the symbol in this figure indicated the resonance frequency of the plane wave defined by Eq.(18). As indicated in this figure, B-type SVU has a lot of resonance frequencies of the plane wave and higher-order mode wave in comparison with those of A-type. Therefore, it can be seen that the soundproofing effect of A-type is better than B-type. 


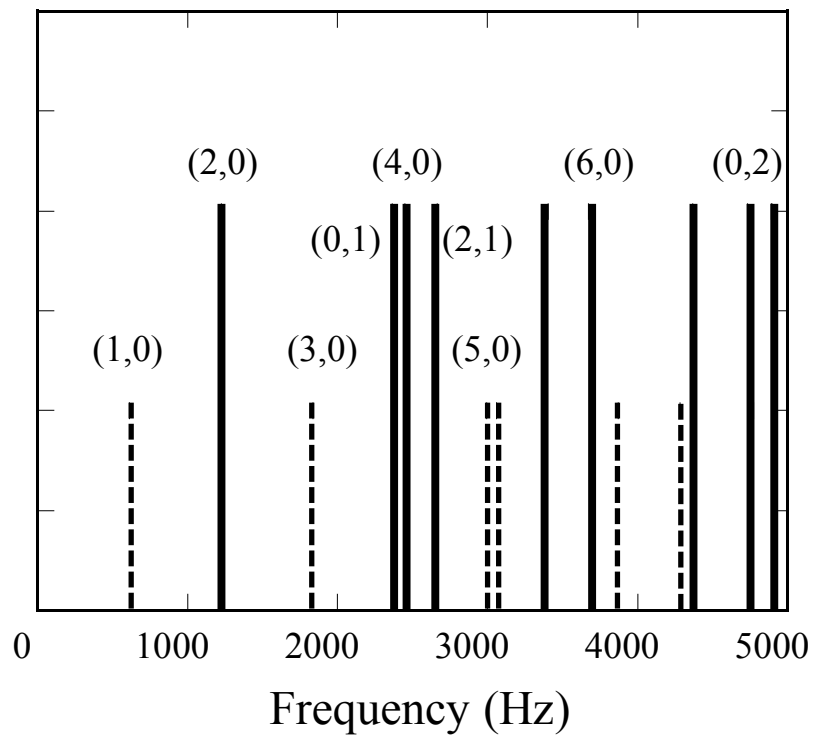

Fig. (5). The first resonance frequency of $(m, n)$ higher-order modes occur in the A-type SVU.

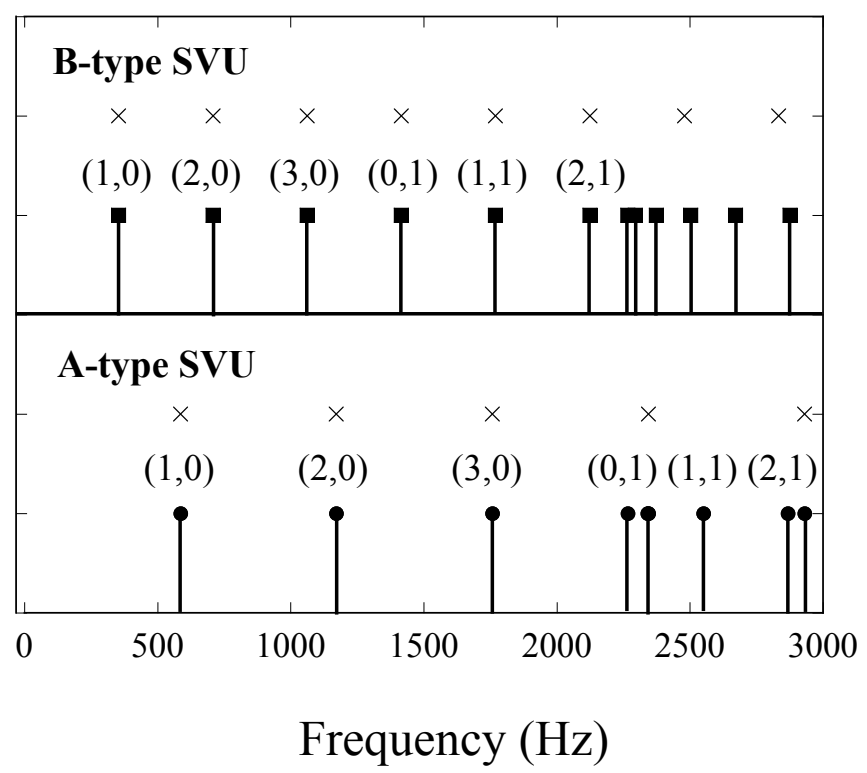

Fig. (6). Comparison of B-type and A-type SVU on the first resonance frequency of $(m, n)$ higher-order modes.

Lastly, in order to increase the soundproofing ability, let us consider the shape and location of inlet and outlet based on the theoretical calculation results. For this purpose, low levels of and in Eqs. (14) and (17) are required. When $a_{i 1}=a / 2-\Delta a, a_{i 2}=a / 2+\Delta a$, and are selected, Eq. (14) becomes

$\bar{Q}_{m, n}^{a}$

$=\left[\frac{2 a}{m \pi} \cos \left(\frac{m \pi}{2}\right) \sin \left(\frac{n \pi}{a} \Delta a\right)\right]\left[\frac{2 b}{n \pi} \cos \left(\frac{n \pi}{2}\right) \sin \left(\frac{n \pi}{b} \Delta b\right)\right]$

This is demonstrated when $m=1,3,5, \ldots$ or $n=1,3,5$, ‥ $\bar{Q}_{m, n}^{a}$ becomes zero. Furthermore, if is selected, for $m=2$, $4,6, \ldots$ also becomes zero. On the other hand, if is selected, becomes zero when $n=2,4,6, \ldots$. Therefore, to avoid many higher order mode waves, the shape of the inlet must be in the form of form-1 or form-2 as shown in Fig. (7). The remaining higher order modes that we have to consider are $(0,2),(0,4),(0,6), \ldots$. When using form- 1 and $(2,0),(4,0)$, $(6,0), \ldots$ when using form-2, respectively. Similarly, a low level of could be selected in an identical manner.
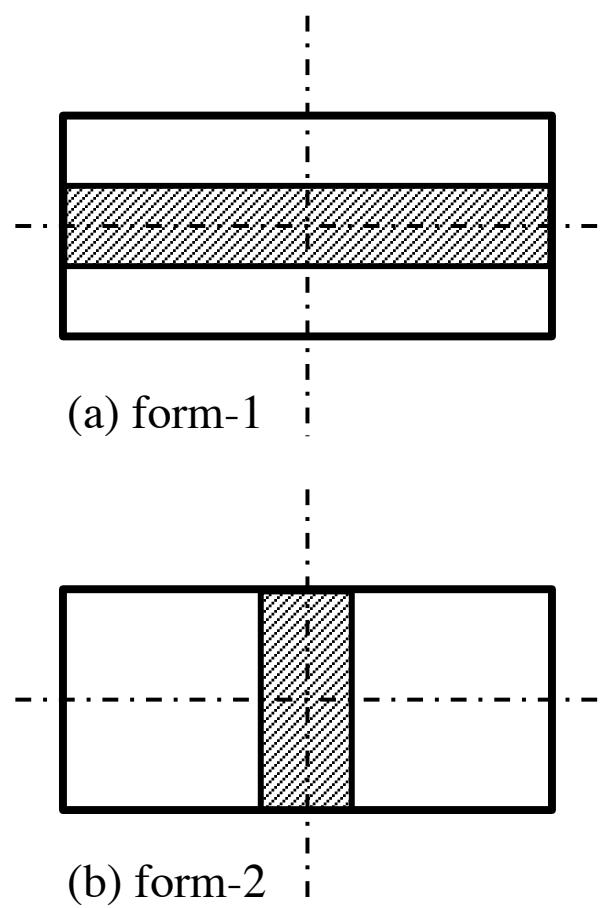

Fig. (7). A reasonable shape and location of the inlet and the outlet which obtained from the analysis for both types.

\section{EXPERIMENTAL RESULTS}

An experiment was conducted using the reverberation chamber method to verify the actual sound attenuation characteristics of A-type and B-type SVU. Fig. (8) shows the experimental setup. The volumes of the reverberation chambers were $98 \mathrm{~m}^{3}$ and $179 \mathrm{~m}^{3}$, respectively. The SVU were installed at the aperture by a casing composed of a plank. The sound source was a loudspeaker fed by pink noise, and five microphones were located on each chamber measuring the spatially averaged sound pressure. The pressure levels in the sending chamber were $100-105 \mathrm{~dB}$ to achieve a pressure level of $70-80 \mathrm{~dB}$ in the receiving room. The actual sound attenuation of the SVU is estimated by the variation from the measurement results with and without the SVU inserted between the two reverberation chambers.

Several SVU with various shapes of inlet and outlet were used during the experiment. The shape and position of inlets and outlets are shown in Fig. (9). Hereafter, we shall refer to them as (A1) to (A5) for A-type SVU and (B1) to (B3) for B-type SVU, respectively. Note that, $\mathrm{S}_{0}$ is an area of inlet or outlet and the symbol $\mathrm{R} \%$ is defined as $\mathrm{R} \%=\mathrm{S}_{0} / \mathrm{S}_{\mathrm{w}}$. Additionally, (A1) (A5), (B1) and (B2) are the shapes that we considered in the theoretical computation and summarized in Fig. (7). The other shapes were used to validate our prediction. 


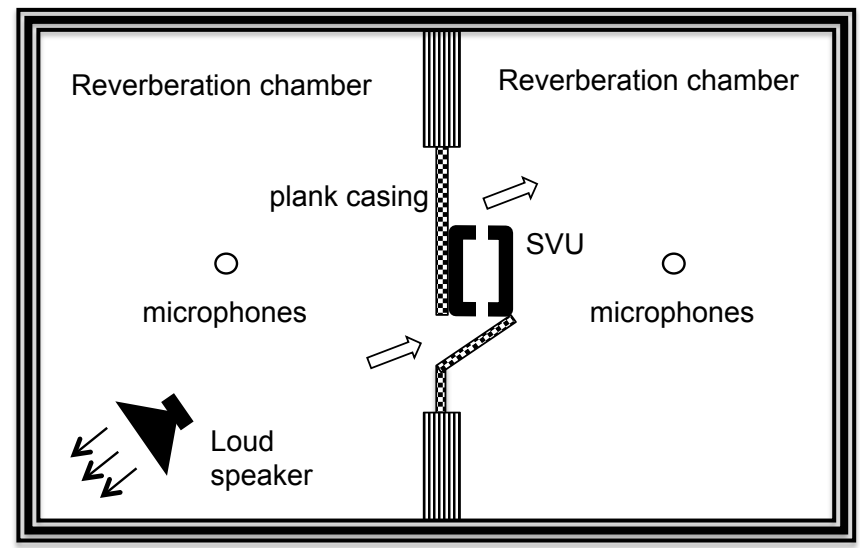

Fig. (8). Measurement system based on the reverberation chamber method.

\section{(A1)}

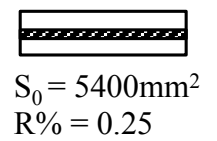

(A2)

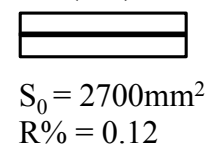

(A3)

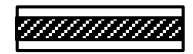

$\mathrm{S}_{0}=10800 \mathrm{~mm}^{2}$ $\mathrm{R} \%=0.5$
(A4)

שU:

$\mathrm{S}_{0}=5400 \mathrm{~mm}^{2}$ $\mathrm{R} \%=0.25$

(A5)

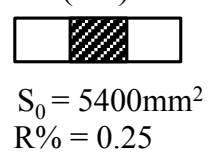

(B1)

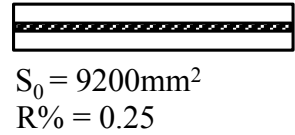

(B2)

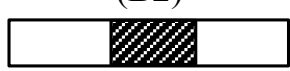

$\mathrm{S}_{0}=9200 \mathrm{~mm}^{2}$

$\mathrm{R} \%=0.25$

\section{(B3)}

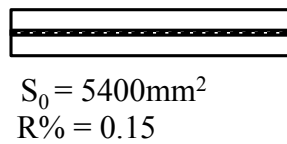

Fig. (9). Combination of inlet and outlet . (A1) to (A5) for A-type SVU with dimension of $75 \mathrm{~mm} \times 290 \mathrm{~mm}$. (B1) to (B3) for B-type SVU with dimension of $290 \mathrm{~mm} \times 480 \mathrm{~mm}$.

Fig. (10) shows the measured results of A-type SVU with changes in outlet cross-section area. Noticeably, the soundproof effect will be decreased when opening a large outlet to maximize ventilation. Similar characteristics are shown in Fig. (11) when changing the inlet cross-section area. Fig. (12) proves our theoretical calculation on the reasonable shape of the outlet as discussed in section 3. Fig. (13) shows the attenuation obtained when using reasonable outlet shapes (A1) and (A5). When using (A5) the attenuation obtained is greater than (A1) because (A5) eliminated the resonance of $(2,0)$ which appears at $1224 \mathrm{~Hz}$. Figs. $(14,15)$ show the difference of A-type and B-type when they have the same $\mathrm{R} \%$ and outlet cross-section area $S_{0}$, respectively. Attenuation obtained is greater when using A-type though B-type has a higher ventilation effect. Lastly, the comparison between two kinds of SVU is shown in Fig. (16) when reasonable shapes of inlet and outlet were used. It is clear that A-type induces greater soundproof effects than B-type.

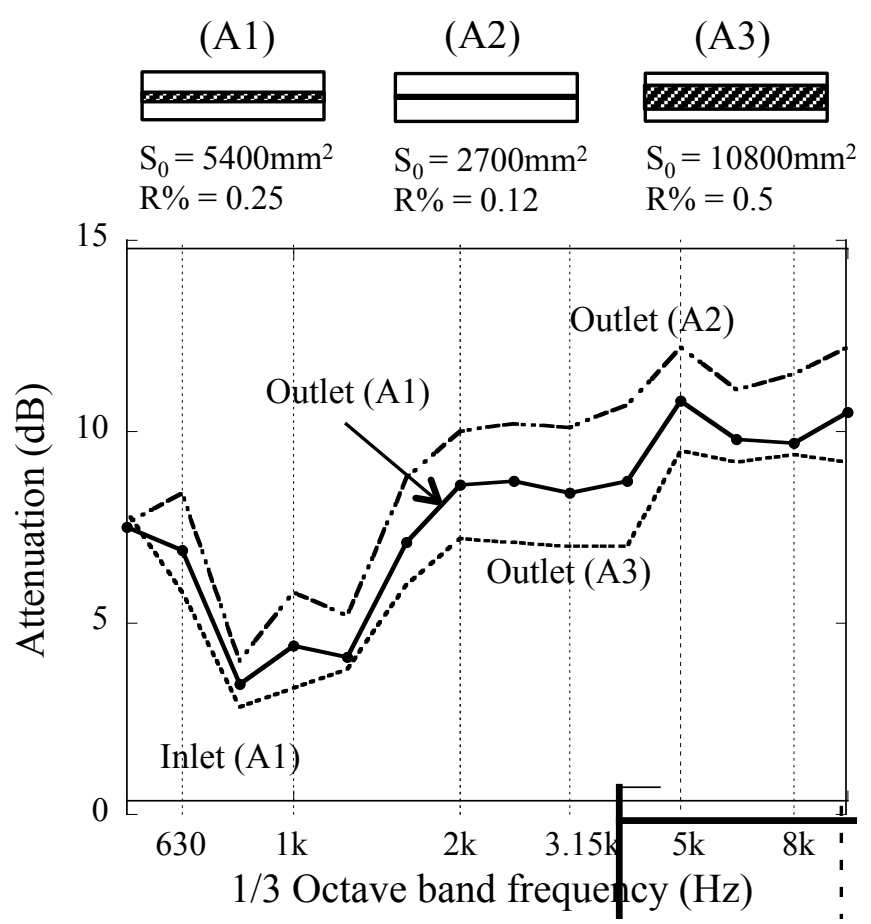

Fig. (10). Measured result of A-type SVU with change of outlet cross-section area.

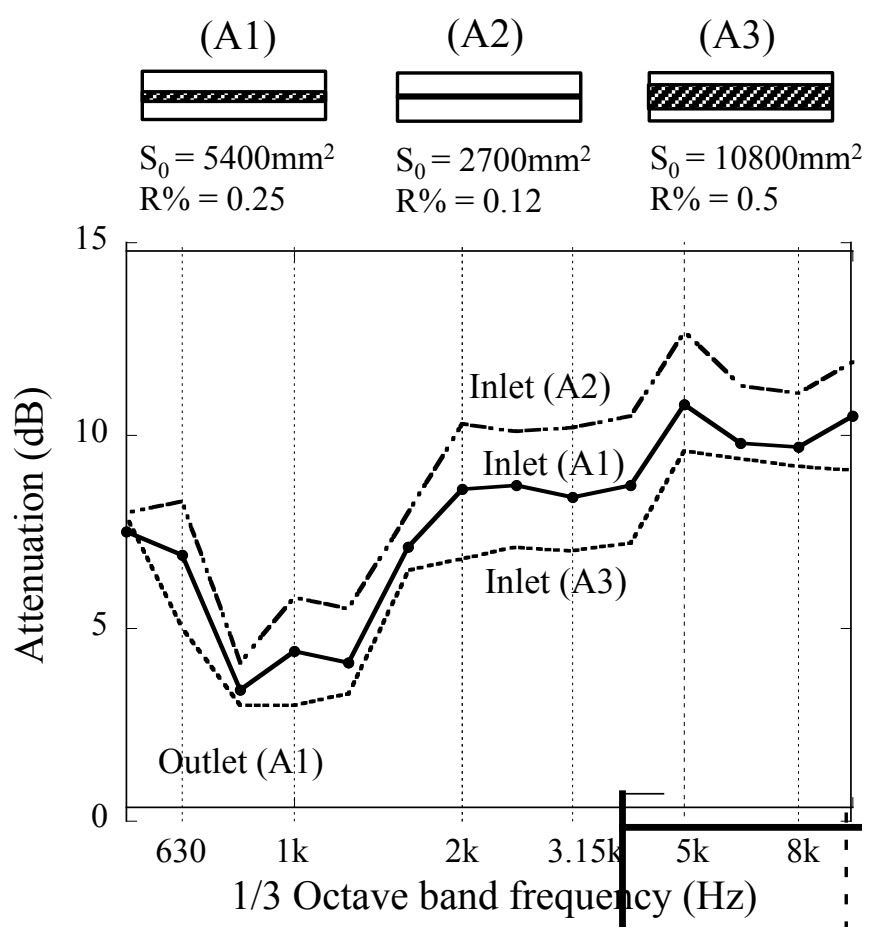

Fig. (11). Measured result of A-type SVU when changing the inlet cross-section area.

It should be noted that the above experiment was carried out using an SVU made of a steel plate that was not affixed with the absorbing material. However, in practice, it is necessary to use some appropriate sound absorbing material inside the SVU to obtain a higher soundproof effect. 


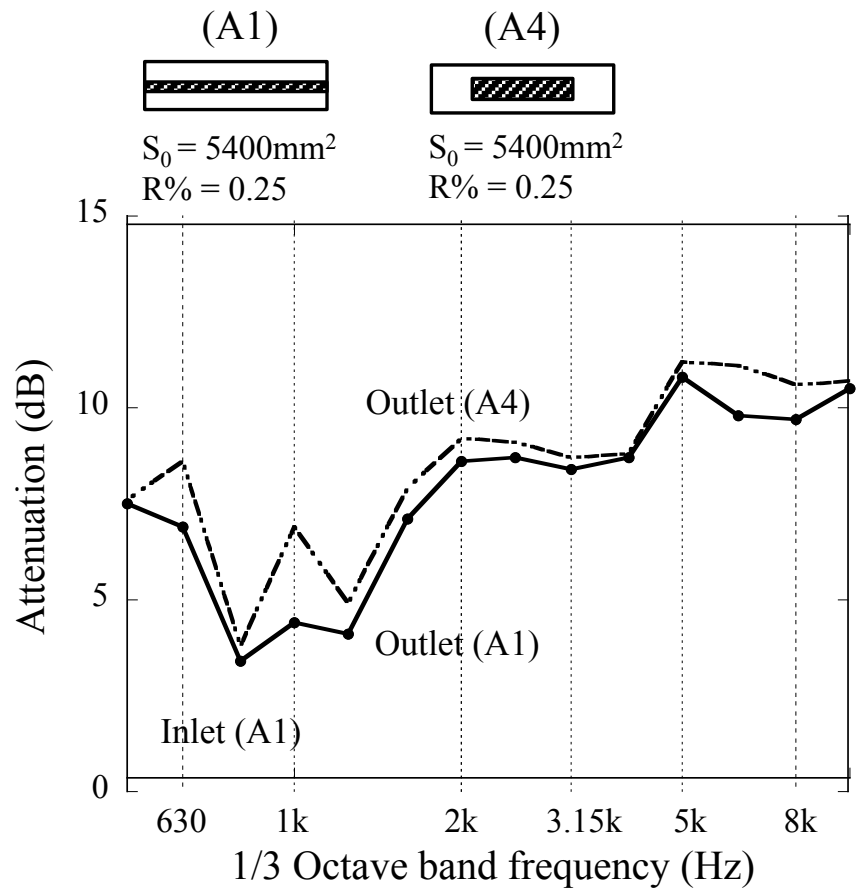

Fig. (12). Measured result of A-type SVU when changing the outlet shape.

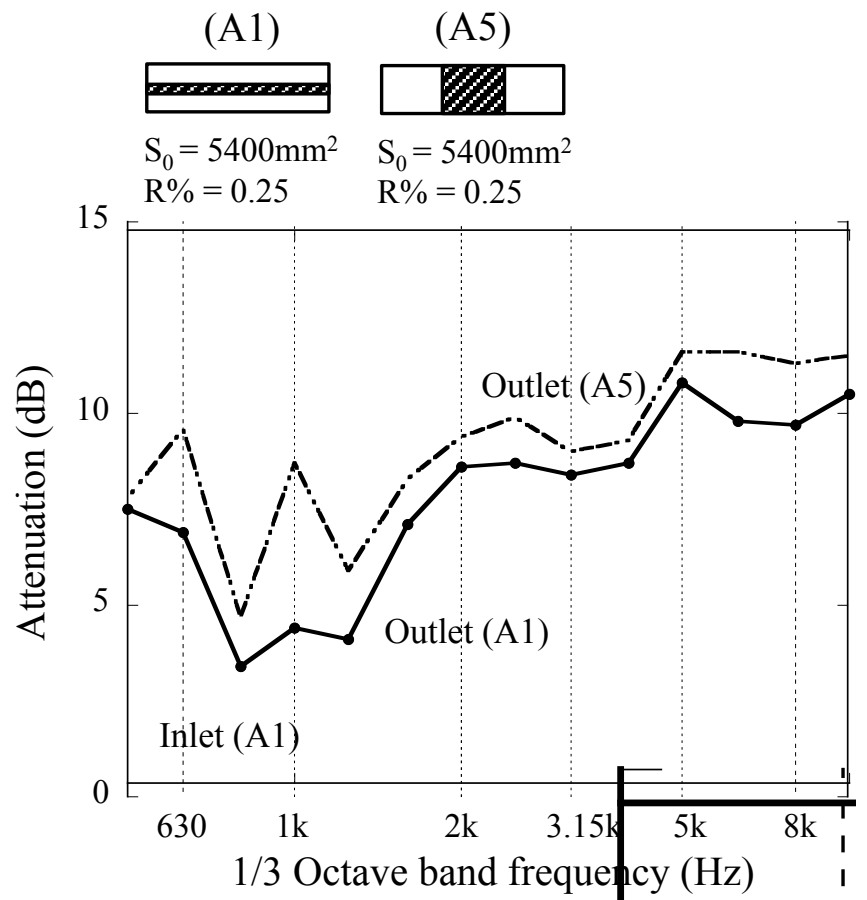

Fig. (13). Measured result when used reasonable shape (A1) and (A5) outlets.

\section{CONCLUSION}

Acoustic characteristics of A-type and B-type rectangular cuboid soundproof ventilation unit have been considered theoretically and experimentally. Both these types have similar dimensions with the inlet and outlet located on the opposite faces. It is considered that B-type would induce better outcomes than A-type because it could open a large input and output to maximize the ventilation effect. However, as various resonances of plane wave and higherorder mode wave occur inside the unit, the soundproofing effect of B-type can not be expected to be great. The results of theoretical calculation and experiments conducted lead to the conclusion that A-type SVU is suitable for soundproof ventilation unit.

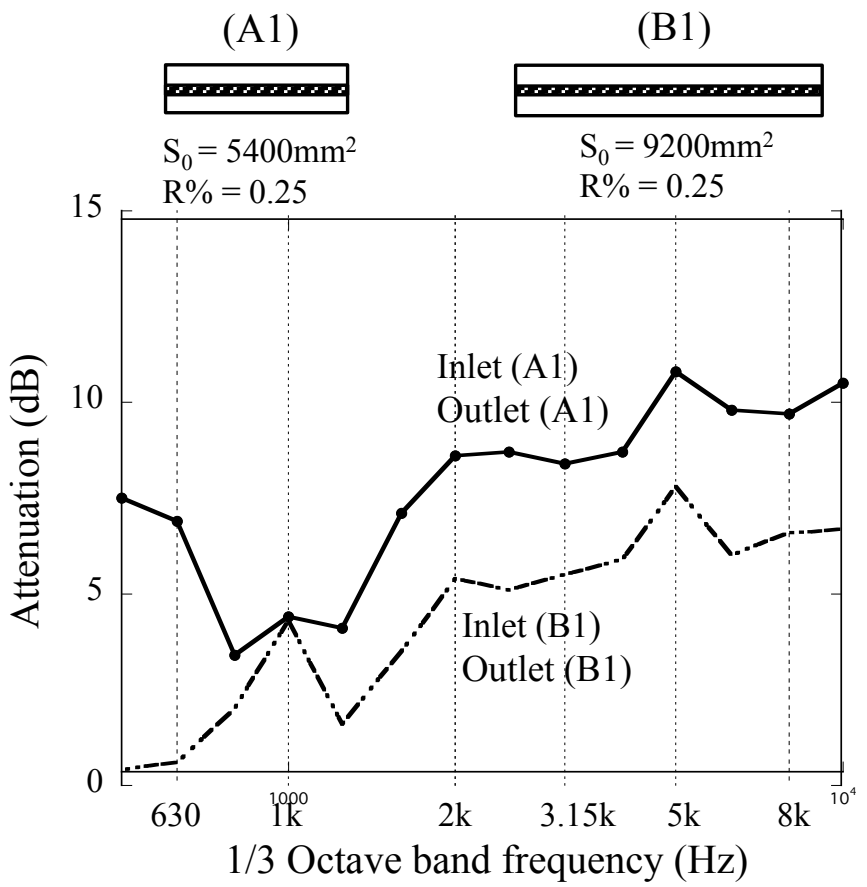

Fig. (14). Measured results of A-type and B-type SVU. Both types of SVU have the same R\%.

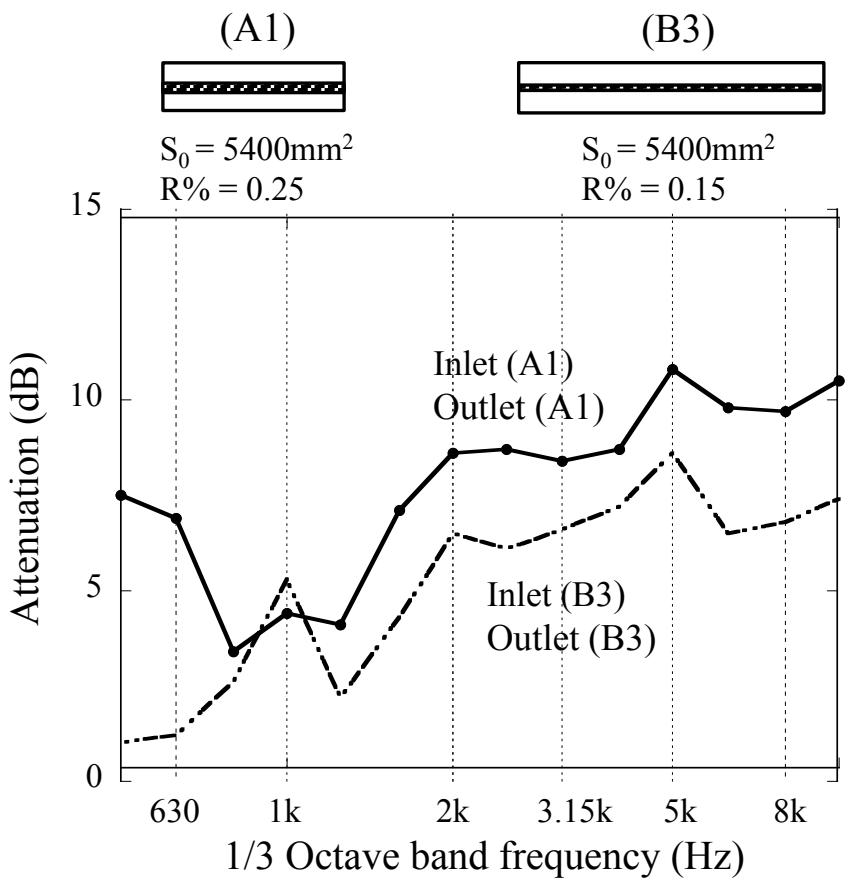

Fig. (15). Measured results of A-type and B-type SVU. Both types of SVU have the same $\mathrm{S}_{0}$ 
(A1)

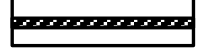

$\mathrm{S}_{0}=5400 \mathrm{~mm}^{2}$

$\mathrm{R} \%=0.25$

(A5)

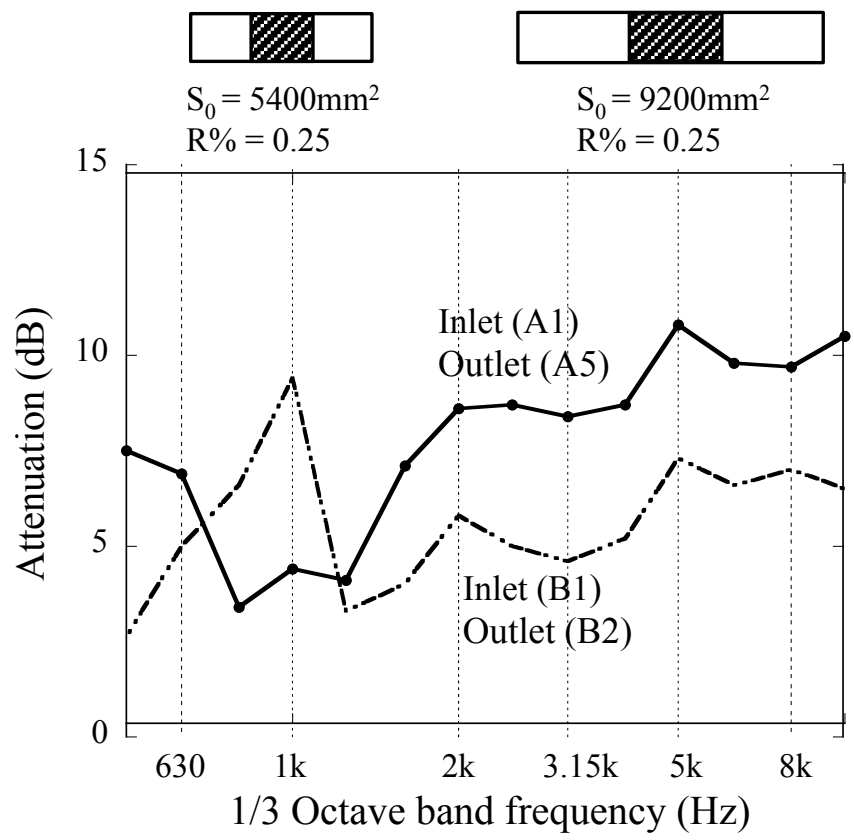

Fig. (16). Measured results of A-type and B-type SVU. Both types of SVU have the same R\% and good combination of inlet and outlet shape.

\section{CONFLICT OF INTEREST}

The authors confirm that this article content has no conflict of interest.

\section{ACKNOWLEDGEMENTS}

Declared none.

\section{REFERENCES}

[1] Nishimura Y, Nishimura S, Nishimura T, Yano T. Sound propagation in soundproofing casement windows. Appl Acoust 2009; 70: 1160-7.

[2] Nishimura S, Nishimura T, Yano T. Acoustic analysis of elliptical muffler chamber having a perforated Pipe. J Sound Vib 2006; 297 : 761-73.

[3] Abom M. Derivation of four-pole parameters for including highermode effects for expansion chamber mufflers with extended inlet and outlet. J Acoust Soc Am 1990;137: 403-18.

[4] Ih JG. The reactive attenuation of rectangular plenum chambers. J Sound Vib 1992; 171:93-122.

[5] Nishimura T, Ikeda T. Four-pole-parameters for an elliptical chamber with mean flow. Electron Commun Jpn 1998; 81: 1-9.

[6] Nishimura T. Ando T. Ikeda T. Resonance of elliptical muffler chamber having a non-uniformly perforated pipe. Electron Commun Jpn 2002; 85: 22-8.

[7] Glav R, Regaud PL, Abom M. Study of a folded resonator including the effects of higher order modes, J Sound Vib 2004; 273: 777-92.

[8] Whalen TM. The behavior of higher order mode shape derivatives in damaged, beam-like structures. J Sound Vib 2008; 309: 426-64.

[9] Carrera E, Miglioretti F, Petrolo M. Computations and evaluations of higher-order theories for free vibration analysis of beams. J Sound Vib 2012; 331: 4269-84.

[10] Nishimura Y, Quang NH, Nishimura S, Nishimura T, Yano T. The acoustic desigh of soundproofing doors and windows. Open Acoust J 2010; 3: 30-7. 